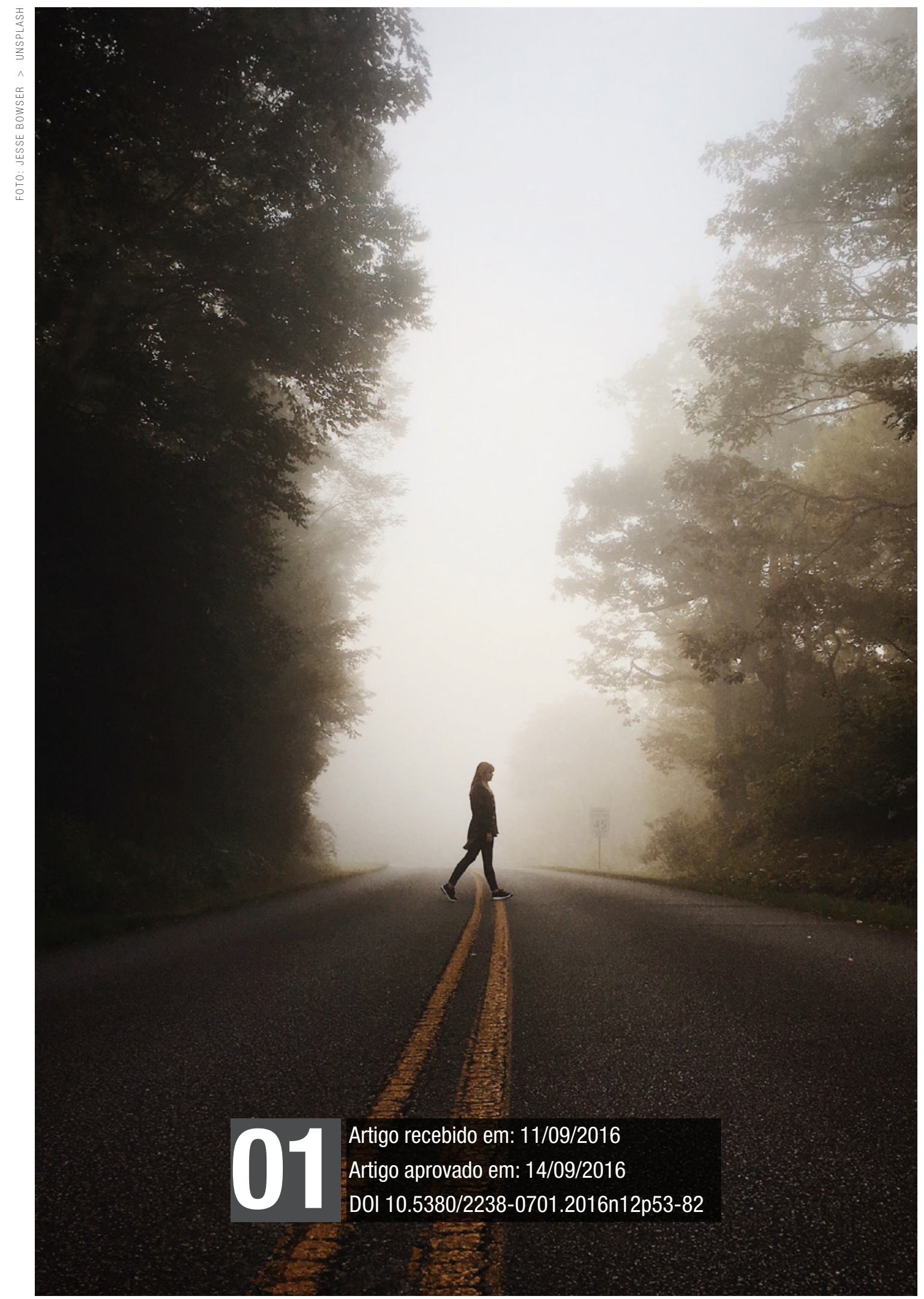


Distância. Formação a distância. Jacquinot. Presença. 


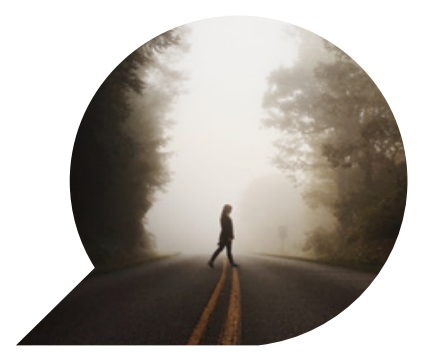

\title{
Distâncias, ausências, proximidades e presenças: conceitos em movimento ${ }^{1}$
}

\author{
Distances, absence, proximités et présences: \\ des concepts en déplacement \\ Distance(s), proximity and presence(s): \\ evolving concepts
}

\section{DANIEL PERAYA ${ }^{2}$}

Resumo: Este artigo apresenta a evolução dos conceitos de distância e de presença em formação à distância tal qual ele se apresenta em duas etapas marcantes: de uma parte o questionamento de Geneviève Jacquinot em 1993 e de outra, os dois números temáticos da revista Distances et Savoirs publicados em 2011 que tentavam responder a mesma questão. Onde vai a distância? Não se trata aqui de uma revisão exaustiva da literatura, mas sim de uma releitura "distanciada" de textos significativos da evolução que levou pesquisadores a pensar a formação à distância a partir do conceito de presença e não mais a partir daquele de distância.

Palavras-chaves: Distância; Formação a distância; Jacquinot; Presença.

1 Tradução de Rosa Maria Cardoso Dalla Costa.

2 TECFA, Université de Genève, daniel.peraya@unige.ch 
Résumé: Cet article présente l'évolution des concepts de distance et de présence en formation à distance telle quelle s'est dessinée entre deux étapes marquantes: d'une part, le questionnement inaugural de Geneviève Jacquinot en 1993 et d'autre part les deux numéros thématiques de Distances et savoirs publiés en 2011 qui tentaient de répondre à cette même question, Où va la distance? Il ne s'agit pas d'une revue exhaustive de la littérature, mais d'une relecture "distancée " de textes significatifs de l'évolution qui a mené les chercheurs à penser la formation à distance à partir du concept de présence et non plus à partir de celui de distance.

Mots-clés: Distance; Formation à distance; Jacquinot; Présence.

Abstract: This article presents the evolution of two key concepts of distance education: distance and presence. These concepts took shape between two landmarks: the first one, the inaugural questioning by Geneviève Jacquinot in 1993 and the second one, two thematic issues of Distances and Knowledge published in 2011 that attempted to answer this question: Where is distance going? This paper is not an exhaustive review of literature, but a "distanced" re-reading of significant texts about the evolution that led researchers to apprehend distance education from the concept of presence and not from the one of distance.

Keywords: Distance; Distance education; Jacquinot; Presence. 


\section{Introdução}

Há aproximadamente 20 anos, Geneviève Jacquinot analisava os desafios da educação a distância diante das mudanças que se anunciavam na área da educação, mas também na área econômica, social e tecnológica: ela se propunha a investigar, no plano pedagógico, o conceito chave da formação a distância, precisamente o de distância, que como sabemos, é antes de tudo, um conceito pedagógico (MOORE, 1993, p. 23). Ela identificou e analisou seis tipos de distância, mostrando como este conceito inicial evoluiu. A questão maior se situa na necessidade de se articular o conceito com certa concepção de tecnologia, mais precisamente da mediação tecnológica que procura "superar a ausência" (1993, p. 64).

A autora retorna a esta temática em 2002 na sua contribuição intitulada "Ausência e Presença na mediação pedagógica ou como fazer circular os signos da presença", no livro dirigido por Roger Guir. Fundamentando-se na análise do uso pedagógico de dispositivos midiáticos (audiovisuais e multimídias educativos, videoconferências, tecnologias digitais e realidades virtuais), ela colocou em evidência a maneira pela qual podemos integrar a presença na distância, ou dito de outra forma como ensinar pode ser "psicologicamente" (1993) ou "mentalmente" (2002) presente mesmo estando fisicamente ausente.

Em 2011, Distances et Savoirs consagrou dois números temáticos para tentar responder à questão "Onde vai a distância? (GUILLEMET et al., 2011a e 2011b), números nos quais podemos descobrir a multiplicidade de novas distâncias (sobretudo DESSUS et al., 2011; FLUCKIGER, 2011, HENRI, 2011), uma tendência à substituição progressiva do conceito de distância em prol daquele da presença a distância (notadamente PERAYA, 2011, KAWWACHI, 2011) ou de proximidades (PAQUELIN, 2011 e 2014), como conceitos chaves da formação a distância.

De todo modo, faz-se necessário conceituar bem a distância além da sua referência como distância espacial: progressivamente emerge e se constrói o conceito de "presença a distância", ou simplesmente de presença em formação a distância, inspirada no modelo anglo-saxão de distância transacional. Um modelo desta presença foi proposto recentemente por Annie Jézégou (2012) no prolongamento de seus próprios trabalhos sobre a distância e a operacionalização da distância transacional (2007). 
Enfim, as numerosas descrições de práticas, em qualquer nível institucional (universidade a distância ou faculdade bimodal) ou individual (dispositivo híbrido de formação), mostram uma diminuição da distinção presença/distância em prol de um processo de generalização da hibridização (sobretudo AUDET, 2011). Notemos, entretanto, que o distanciamento das atividades pedagógicas no quadro das formações superiores presenciais constitui uma das características que definem os dispositivos híbridos de formação (CHARLER et al., 2006; PERAYA et al., 2014): esses dispositivos partilham (dividem) com a educação a distância (EAD) certas características e demandam, portanto, a mesma reconceitualização em termos de presença.

Nesta contribuição, tenho como proposta utilizar alguns destes textos significativos para descrever os deslocamentos que ocorreram da distância à presença e apresentar o estado atual dessas conceitualizações. Não se propõe aqui a produção exaustiva da literatura. Inserida na temática deste número especial, este artigo é parcial: procura apresentar a evolução de alguns conceitos chaves aos quais Jacquinot antecipadamente e com muita propriedade contribui e a propósito da qual Distance et Savoirs procurou aprofundar nos seus dois números temáticos de 2011. Excepcionalmente vou inserir a partir de outros autores ${ }^{1}$ argumentos que se inscrevem na reflexão e que, à sua maneira, aprofundam-na e a atualizam.

\section{Uma cartografia das distâncias}

\section{Da Distância às Distâncias}

A característica fundadora da EAD, afirma Jacquinot, é a distância, instalada (nichée) no coração da relação pedagógica. Com efeito, a vontade de escolarizar um público impedido de frequentar as instituições de ensino, ou dito de outra forma, uma maneira de vencer a distância e levar o "conhecimento à domicílio" (HENRI; KAYE, 1985), constitui o primeiro objetivo da EAD. A educação a distância se define assim em oposição à educação presencial, pela ruptura ${ }^{2}$ de co-presença espacial entre o educador e seus alunos: "o professor e

10 masculino é utilizado no presente artigo sem discriminação e unicamente com o objetivo de deixar o texto mais leve.

2 Eu amo dizer, de forma provocativa, que a formação presencial é marcada por um destino "dramático". Não é ela em efeito, à imagem do teatro clássico regida por três unidades: unidade do tempo, do local e da ação? 
o estudantes estão distantes" (op. cit., p. 10). Quanto à ruptura temporal, ela é indissociável da distância espacial, da qual ela é consequência: "as atividades de ensino e de aquisição de conhecimento são aí separadas no tempo e no espaço, afirma Viviane Gilkman (2002, p.13). Esta dupla distância foi considerada durante muito tempo e está ainda presente em alguns meios como fundamento da $\mathrm{EAD}^{3}$ com suas consequências: "esta definição enrigeceu o quadro de práticas como aquele da análise, conservando uma visão de modalidades organizacionais de formação reduzida à alternância, seja em presença, seja a distância” (PERAYA, 2011).

Em seu artigo de 1993, Apprivoiser la distance et supprimer l'absence? ou les défis de la formation à distance, Jacquinot identifica diversas distâncias - geográfica, temporal, tecnológica, sociocultural, sócio-econômica e pedagógica - todas consequências dessa ruptura inicial no centro da relação pedagógica. A autora analisa seus aspectos essenciais e sua complexidade - entre vantagens e desvantagens - e mostra as dificuldades de identificá-las: cada uma parece, com efeito, ter um valor agregado potencial, resultado de discursos prescritos, que comprometem a qualidade do processo de ensino e de aprendizagem como dispositivo de formação, mas que na realidade, induzem a efeitos contraditórios e até mesmo contraprodutivos.

As distâncias espaciais e temporais permitem ao aprendiz ter acesso aos recursos o tempo todo e em todo lugar: este é sempre um dos argumentos mais difundidos em favor do e-learning, particularmente pelas empresas multisites que vêm nesta modalidade de distribuição do ensino uma maneira de reduzir de maneira drástica o custo global, evitando o deslocamento de seus funcionários para um centro de formação, na sede da empresa. Portanto, "os públicos com menos escolarização que têm mais dificuldade de gerenciar seu trabalho pessoal" (JACQUINOT, 1993, p. 57) seriam prejudicados por esta modalidade de organização da formação. Nesta época, já sabíamos que “a

3 Notemos que essa distinção foi relativizada pelos trabalhos de Cropley e Kahi (1983) que citam Henry e Kaye (1985, p. 16. Todos os dispositivos de formação mesmo aqueles reconhecidos classicamente como presenciais, comportam uma de trabalho a distância: a presença de um aluno ou de um estudante da formação (a classe, o anfiteatro, o laboratório, etc) como a copresença dos aprendizes e do professor não constituem que um momento de aprendizagem e de construção de conhecimentos pelo aprendiz. Uma parte importante desses processos é, de fato, feita pelo estudante, sozinho ou com a ajuda de seus próximos, de seus pares ou de todo tipo de ajuda externa, em sua casa, na biblioteca, etc, mas em todo caso fora da co-presença do professor. Entre as formações a distância, híbridas e presenciais, a distância espaço-temporal será portanto, uma questão de grau, de importância relativa. 
gestão dessa distância temporal entre as demandas do aprendiz e as respostas da instituição [...] constitui um dos fatores fundamentais da qualidade do enquadramento" (JACQUINOT, ibidem).

É por isso que é necessário multiplicar os encontros presenciais, em detrimento dessa liberdade e da flexibilidade possibilitada pela educação a distância. Retornamos aqui a um dos limites da formação parcialmente ou inteiramente a distância nas suas formas convencionais: a flexibilidade e o assincronismo sobre os quais se constroem numerosos projetos se encontram em contradição com os princípios dos reagrupamentos sincrônicos, percebidos como avanço de qualidade dessa modalidade e como uma condição de êxito dos aprendizes. Isso é verdadeiro, tanto para os reagrupamentos que ocorram na presença - in locu - ou em telepresença através de um dispositivo de comunicação mediatizado num ambiente digital.

Tomemos um exemplo, aquele das distâncias socioculturais e socioeconômicas. O projeto educativo sobre o qual se desenvolvem inúmeras universidades a distância ditas "abertas", na linha do CNED e da Open University (universidde aberta) era aquele de um ensino da segunda oportunidade: oferecer uma possibilidade de formação superior e universitária a um público excluído do sistema educativo no seu percurso em formação inicial. Ora, aponta a autora, esta distância, que deve ser compreendida no sentido de uma primeira ruptura, é bem difícil de ser superada: a universidade aberta inglesa, nascida num projeto político trabalhista em 1965, permitiu, sobretudo "a reciclagem de pessoas que tinham um certo nível universitário, aos professores notadamente" (JACQUINOT, 1993, p. 59).

As enquetes relativas ao desenvolvimento dos MOOCs, "os zombadores", são titulares de um diploma de ensino superior (Pomerol, 2014). Nos Estados Unidos, uma pesquisa recente feita pela Universidade da Pensilvânia ${ }^{5}$, apresenta resultados ainda mais significativos: $83 \%$ dos participantes dos MOOCs são titulares de um diploma de curso pós-médio de 2 a 4 anos (CHRISTENSEN et al., 2013).

A distância pedagógica enfim é aquela que surge entre aquele que "deve ou quer aprender e aquele que sabe e quer ou deve apren-

4 Podemos pensar que se trata de dados que dizem respeito aos MOOCs franceses. 0 documento consultado não o diz.

5 A enquete se baseia em 32 cursos disponíveis sobre a plataforma Coursera e de vários países divididos em quarto grupos: Estados Unidos (34\%), Países que não pertencem à OCDE (31\%), BRICS (Brasil, Rússia, India China e África do Sul, 14,8\%), outros países desenvolvidos. 
der” (JACQUINOT, 1993, p. 60). Citando Michel Serres, que denominou "uma universidade sem distância" (1992, apud JACQUINOT, ibidem), tratava então de reduzir "todos os distanciamentos possíveis entre as fontes do saber e do conhecimento e aqueles que desejavam aprender e deles se apropriar" (ibidem). Esta dupla definição da distância pedagógica nos remete aos dois eixos do triângulo pedagógico de Jean Houssaye (1988): o primeiro liga o professor ao aprendiz, ou o eixo do ensino, enquanto o segundo liga o aprendiz e as fontes de conhecimento, que eu reinterpreto em termos de recurso, incluindo os professores: é o eixo da aprendizagem. Esta distância, destaca Jacquinot, parece a mais difícil a reduzir. Sem dúvida porque todas essas distâncias estão interligadas (TROPE; LIBERMANN, 2010, apud DESSUS et al., 2011, p. 474) e que a diminuição da distância pedagógica depende globalmente da redução de cada uma delas.

\section{Outras distâncias, ainda}

Didier Paquelin (2001b), de quem comentarei as proposições abaixo (cf. 2.2), propõe sucintamente duas outras formas de distância: as distâncias cognitiva e pragmática que, do meu ponto de vista, especificam a distância pedagógica identificada por Jacquinot. A primeira "ligada à compreensão dos conteúdos" (op. cit., p. 566) enquanto que a segunda "é ligada à capacidade de colocar em prática as teorias e de formalização de problemas concretos” (ibidem). O autor nos remete assim ao eixo da aprendizagem e o define em termos de cognição e de domínio dos processos congnitivos.

As pesquisas de Cédric Fluckiger, inspiradas nos trabalhos de Rabardel, tratam dos processos de iniciação instrumental principalmente entre os jovens, colegiais e estudantes. O autor consagra sua contribuição ao primeiro volume de Où va la distance? (2011a) à emergência dos ambientes digitais institucionais universitários e pessoais desenvolvidos pelos estudantes. Ele constata, baseado em estudos empíricos exploratórios, que

[...] os estudantes, enquanto que jovens adultos, dispõem de uma grande gama de instrumentos de comunicação interpessoal e de acesso à informação (CREDOC, 2009), assim como de hábitos de uso profunda e longamente estabelecidos [...]. Entretanto, se os es- 
tudantes comunicam bastante em geral, eles o fazem bem menos no meio educativo: eles são grandes utilizadores da web 2.0 e de redes sociais, mas as pesquisas apontam a constatação recorrente de dificuldades de integração da web 2.0 e mais genericamente de formas colaborativas e participativas de ensino a distância no superior. [...] (FLUCKIGER, 2011, p. 398-399).

Notemos que a literatura faz a mesma constatação a propósito dos professores que têm dificuldades para transferir suas competências tecnológicas obtidas na esfera de suas atividades pessoais e privadas à sua prática pedagógica. Eu aproximaria essas novas formas de distância cuja emergência encontra sua origem nas tecnologias web 2.0 à distância tecnológica inicialmente definida por Jacquinot em termos de fratura (ou ruptura) digital do primeiro nível, aquela da acessibilidade às tecnologias e, em seguida, da adaptação e da integração dessas às necessidades específicas da pedagogia em EAD.

Hoje, considerando o grau de generalização do acesso às tecnologias nos países desenvolvidos, a distância tecnológica que estuda Fluckier releva do segundo nível dessa fratura digital, aquela dos usos de tecnologia eles mesmos, assim como o tratamento da informação às quais ela dá acesso. Essas distâncias entre os usos comunicacionais ordinários dos estudantes e aqueles que o meio acadêmico sonharia ver se desenvolver inícios instrumentais diferentes. Esta tese da "descontinuidade instrumental" rompeu com uma corrente 'continuista' que defende, quanto a ela, a tese da contradição dos usos graças a "objetos fronteiriços" ou objetos contrabandeados de usos presentes em diferentes esferas de atividades, pessoais, profissionais, acadêmicas (FLUCKIER, 2014). Pois como é necessário bem nomear as coisas para que elas possam existir em nosso entendimento, eu proporei chamar essa distância, uma distância instrumental.

France Henri (2011) na sua contribuição ao segundo volume de Où va la Distance? (2011b) propõe uma releitura de alguns do meus trabalhos ${ }^{6}$ neste sentido (PERAYA, 2008, 2012). Trata-se para mim, nestes dois artigos, de estabelecer, a partir dos trabalhos de JeanFrançois Luotard e da oposição entre os saberes científicos e narra-

6 Esta reflexão nasce no âmbito da comissão "éthique-plagiat" da Reitoria da Universidade de Genebra na qual o objetivo era o de analisar o fenômeno do plágio nos trabalhos universitários. Eu defendi uma abordagem descritiva e compreensiva argumentando que este fenômeno não revelava necessariamente uma vontade de fraude, mas uma transferência de práticas comunicacionais e informacionais pessoais na esfera acadêmica, as quais não podem ser consideradas como legítimas pois as regras pragmáticas são diferentes. 
tivos (1979), um quadro de análise da influência das tecnologias e particularmente daquelas da web 2.0 sobre a cultura informacional dos estudantes, sobre o processo de produção e de difusão de conhecimentos. Eu formulei, como hipótese $\mathrm{e}^{7}$ que as práticas comunicacionais próprias das redes sociais (MySpace, Ning, Facebook, etc) e dos blogs, que os usos e instrumentos de partilhamentos de documentos e de meios (textos, músicas, fotos, vídeos, etc.) e de indexação (folksonomy), de agregação, de estruturação, ou de partilhamento em linha de recursos (referências, ligações, web) etc., induziriam práticas de produção de conteúdo (notadamente le remixing) e de sua difusão próxima dos saberes narrativos. Os estudantes importariam de tais práticas, em ruptura com as regras de validação dos saberes científicos, no centro de seus trabalhos universitários:

Sempre segundo Peraya, os estudantes que utilizam a web social importariam das suas práticas pessoais de comunicação próprias à construção de saberes narrativos, deslocando assim as práticas legitimadas no meio acadêmico que sustentam a elaboração do saber cienteífico. [...] (HENRY, op. cit, p. 621).

O aparecimento das tecnologias da web 2.0 e dos usos a ele ligados, sugerem, portanto, segundo o autor, uma nova distância que eu denominarei epistemológica (sentido 1) na medida em que esses dois tipos de saberes revelam concepções epistemológicas diferentes. Entretanto, minhas análises, não consideram a EAD nem como objeto nem como terreno. Trata-se então de uma aplicação interessante de um quadro de análise geral dos efeitos induzidos pelo uso das tecnologias no âmbito da formação à distância.

Enfim, na sua tese recente, Clément Dussarps (2014, p.138) sugere a existência de uma distância financeira que nós poderíamos relacionar à distância sócio-econômica identificada por Jacquinot (1993). Esta distância dá conta da dificuldade ou impossibilidade do aprendiz de se inscrever em uma formação aberta à distância. Nós poderíamos precisar os diferentes componentes desta distância notadamente em relação aos custos ocasionados, diretos (taxas de inscrição) ou indiretos (taxas de conexão, por exemplo). Esta dificulda-

7 Esta hipótese deu início a dois projetos de pesquisa empírica feitos em colaboração com Bernadette Charlier (Universidade de Fribourg) e de colegas das faculdades de letras de uma parte, de tradução e interpretação de outra parte da Universidade de Genebra. Estes projetos infelizmente não receberam os financiamentos pedidos. 
de é bem real. Eu cito a título de exemplo o projeto de aumento da contribuição própria dos estudantes às taxas de inscrição, estudantes em sua maioria oriundos de países do Sul e bolsistas da AUF: esta medida prevista para o início do ano escolar de 2015, se ela fosse aplicada em sua forma inicial, teria tornado inacessível a formação de inúmeros estudantes, sobretudo aqueles que desejam seguir a formação Acrédité ${ }^{8}$ qual TECFA participa desde a criação.

\section{A Distância como ruptura: a medida do qualitativo}

Na introdução de seu artigo já mencionado, Paquelin (2011) distingue oito formas diferentes de distância: aquelas mencionadas por Jacquinot mais as distâncias cognitiva e pragmática. Ele notava ainda que "alguns tipos de distância tem uma separação, características que nenhuma métrica poderia atualmente definir” (p. 566). Ele considerava impossível operacionalizar a medida dessas distâncias numa escala quantitativa. Assim a única abordagem dessas distâncias para ele, parece ser a qualitativa: a distância não deve ser nem tão significativa nem tão frágil: apenas uma "boa distância" (ibidem), repleta de proximidades, permite manter a eficiência do dispositivo.

Alguns pesquisadores adotaram outra direção. Seus enfoques assumem a distância como uma separação e a tornam objectivável, ou seja, mensurável. É essa a pista seguida por Philippe Dessus e seus colegas nos seus trabalhos apresentados no volume 2 de Où $v$ ala distance? (DESSUS et al., 2011). Do ponto de vista desses autores,

as diferentes teorias sobre a distância [...] não comportam sempre critérios mensuráveis a não ser que consideremos raras tentativas de tornar quantificáveis a distância de transação. [...] (DESSUS, 2003; SABA; SHEARER, 1994, p. 474)

Seus trabalhos consideram como ponto de partida a distância cognitiva e/ou epistemológica (sentido 2): "existe uma distância necessária entre o que sabe o professor e o estudante" (op. cit., p. 474). Para evitar qualquer confusão com a distância epistemológica (sentido 1), eu considerarei neste texto a denominaçãoo de distância cog-

8 Esta formação às tecnologias educativas e ao desenvolvimento de dispositivos inteiramente ou parcialmente à distância (antigamente UTICEF) é organizado conjuntamente pelas Universidades de Cergy-Pontoise de Mons e de Genebra. Ver: http://foad.refer.org/rubrique16.html 
nitiva que poderá se inscrever no sentido de distância pedagógica para Jacquinot ou no de distância congnitiva proposta por Paquelin. Eu o farei, pois esses dois termos não parecem estar definidos explicitamente por esses autores.

Oriundos do campo da $\mathrm{EIAH}^{9}$, seus trabalhos visam "dar conta da distância não em metros ou com cronômetros, mas através de instrumentos automáticos da língua" (ibidem). A recente integração dessas ferramentas nas plataformas de ensino a distância apresentam respostas a questão sobre que tipos de distância eles podem fazer surgir para em seguida medi-los. Dessus e seus colegas descrevem a experiência - para mais detalhes sobre isso indico a leitura do artigo - de três widgets, de três aplicações, inseridas na plataforma de educação distância: Pensum, CONSPECT e PolyCafe. Essas aplicações permitem objetivar, respectivamente a) uma "distância semântica" intratextual e portanto as incoerências internas das produções textuais dos estudantes; b) uma "distância cognitiva individual" entre as diversas produções de um estudante no tempo ou com aquelas de outros autores e enfim, c) uma "distância cognitiva social" que analisa por sua vez as falas nos dispositivos de comunicação mediatizados e que dão conta do peso de cada um nas interações de grupo e nos processos de colaboraçãoo (op. cit., p. 477).

\section{Proximidade e Presença à distância}

A presença das ausências:

interatividade intencional, intransitiva e transitiva

Para Jacquinot, a ausência é correlacionada à distância: distância e ausência são as duas faces da relação pedagógica em EAD. A lei de 12 de julho de 1971 insiste sobre essa ausência: "o ensino a distância é um ensino que não compreende, nos locais onde ele é recebido, a presença física do professor encarregado de transmitir, ou não comporta sua presença a não ser de forma ocasional e para uma certa atividade" (apud GLIKMAN, 2002, p.13). A AFNOR ${ }^{10}$ não diz outra coisa: um dispositivo de formação a distância é "um sistema de for-

9 Environnements Informatiques pour l'Apprentissage Humain - Ambiente informático para o aprendizado humano (Nota da tradutora).

10 A Associação AFNOR e suas afiliadas constituem um grupo internacional a serviço do desenvolvimento econômico das organizações (Nota da tradutora). 
mação concebido para permitir aos indivíduos de se formar sem um deslocamento para um lugar de formação e sem a presença física de um formador" (apud PAQUELIN, 2011, p. 569).

Nos textos citados, Jacquinot chama a atenção para que se considere e se trate a distância não como um 'vazio a ser vencido' como um "defeito - a ausência - que precisa ser vencida", notadamente graça às tecnologias" (JACQUINOT, 1993, p.64), "projeto perpetualmente decepcionante pelo excesso de ausência como de presença" (2002, p. 105). A videoconferência, como é classicamente utilizada, resolve paliativamente a falta de presença e se inscreve assim numa lógica de decepção. Nesta perspectiva, notamos que as teorias da riqueza dos meios (Daft; Lengel, 1987; Dennis; Valacich, 199; CLARKE; BRENNAN, 1991) que postulam que todos os meios não têm um potencial de comunicação idêntico são frequentemente evocadas no âmbito de pesquisas relativas à formação e à colaboração mediatizada. Mas todos usam como referência ou por comparação a situação da comunicação presencial face a face. Não é por esse caminho que Jacquinot se propõe a explorar, pois superar a ausência mais que a distância não mudaria fundamentalmente nada em termos de conceptualização a não ser ao nível das práticas pedagógicas.

Ela propõe ao contrário "apprivoiser la distance" o que significa conceber a EAD, a partir da noção de presença e mostra, a partir de exemplos concretos (audiovisuais e multimídias educativos, videoconferência notadamente), que um enfoque comunicacional da relação pedagógica mediatizada, permite fazer circular nos dispositivos mediatizados os sinais da presença do professor - o polo de emissão da relação de comunicação e um estudante ideal - o polo de recepção do processo comunicacional - mesmo na sua ausência física. Em consequência, o autor (o expert, o professor, etc) e o estudante podem se tornar presentes um face ao outro num dispositivo comunicacional. O termo dispositivo comunicacional e de formação mediatizada, que eu frequentemente propus, encontra aqui seu valor heurístico.

Eu analisarei um desses exemplos, o das emissões ou filmes educativos, que me parece o mais original: ele se fundamenta nos conceitos de interatividade intencional (BARCHECHATH; POUTS-LAJUS, 1990) de uma parte, de outra intransitivo (CHÂTEU, 1990, citado por JACQUINOT, 2002, p. 107) ou transitivo (notadamente JACQUINOT, 1997) que parece infelizmente pouco in- 
teressante para os pesquisadores atualmente ${ }^{11}$. A interatividade intencional se situa no polo da emissão: ela diz respeito a parte do dispositivo (na origem o conceito nasce no contexto do software educativo) que estabelece e organiza o protocolo de comunicação entre o utilizador e o autor. $\mathrm{O}$ autor está obviamente ausente fisicamente no momento da apropriação do dispositivo pelo estudante, mas ele inseriu no dispositivo formas de comunicação e de interpelação, marcas de enunciação, destinadas ao estudante ao qual ele se dirige: a interatividade intencional manifesta a vontade do autor de inserir, implicar o estudante no processo comunicacional; também o insere nos protocolos de comunicação interpessoal. Ao mesmo tempo, Moore, fundamenta seu modelo de distância transacional sobre a existência de um diálogo, mesmo que virtual, entre o professor e o aluno através dos meios (1993, p. 25).

Quanto à interatividade intransitiva (relativa aos meios tradicionais) e transitiva (relativa aos meios digitais interativos), elas destacam principalmente o polo da recepçãoo à medida que elas objetivam transformar o estudante cognitivamente ativo um 'espectador', tido como um real ator dos dispositivos interativos. A cenarização solicita do estudante "uma atividade sensorial, afetiva e intelectual a serviço da interpretação da mensagem" (CHÂTEAU, 1990, citado por JACQUINOT, 2002, p. 107). Essas diferentes formas de interatividade estão próximas de um contrato de leitura ou de um contrato enunciativo que exprime a maneira como um enunciador se mostra através das escolhas que ele faz ao colocar em prática os materiais textuais que compõem o dispositivo.

Eu não me alongarei mais sobre esta contribuição sobre os conceitos, apesar do seu grande interesse: eles mostram, em relação aos quadros teóricos da época, como os processos de mediatização assim como a cenarização permite tornar presente um ao outro, o autor e o utilizador, o emissor e o receptor, o professor e o estudante num dispositivo de comunicação e de formação mediatizado. $\mathrm{O}$ fato des-

11 Ele está de acordo com o conceito de suplantação das operações mentais proposta por Salomon (1979) que designa o fato, para um meio de representar visualmente e explicitamente uma operação a fim que o aprendiz a internalize. Em 2010, interrogado par B.Charlier a propósito da perenização deste conceito ele respondeu que ele não era utilizado pelos pesquisadores por uma razão 'surpreendente' pois "os pesquisadores não vão querer olhar para trinta ou quarenta anos atrás" (2010, p.33). Jaquinot, Albéro e eu mesmo assinalamos esta tendência própria no sentido de não se ter consciência da história do nosso domínio, como se cada "última tecnologia" (Jacquinot, 1998) apresentasse problemas inteiramente novos. 
tas formas de interatividade se basearem essencialmente em aspectos cognitivos do processo e secundariamente sobre aspectos relacionais, pois comunicar é também colocar interlocutores em relação, constitue, sem dúvida seu limite (MEUNIER; PERAYA, 1993). Hoje em efeito, essas são todas as dimensões de um dispositivo em formação que permite mediatizar as tecnologias (PERAYA, 1999 e 2010). Mas em todo caso, essas formas de interatividade constituem um ponto de vista essencial entre "a distância que separa e a proximidade que liga” (PAQUELIN, 2011, p. 566), o ponto de partida em direção a um novo paradigma, aquele da presença na distância.

\section{Proximidade e Presença na distância}

\section{No despertar da distância internacional: do lado das presenças}

Paulo Kawachi (2011) lista, depois estrutura as diferentes e numerosas acepções do termo presença que aparecem na literatura referente à comunicação mediatizada pelo computador e a educação a distância. Dentre todas elas, ele destacará seis: a presença institucional $^{12}$, a presença do aprendiz ${ }^{13}$, a presença cognitiva ${ }^{14}$, a presença social ${ }^{15}$, a presença transacional ${ }^{16}$, e enfim, a teaching presence ${ }^{17}$. O autor distingue e conserva essas diferentes presenças porque elas são

12 A presença institucional é definida como o capital sociocultural e acadêmico da instituição, sua reputação, etc.

13 A presença do aprendiz definida como o fato de considerar o aprendiz como "uma pessoa real, através de suas interações on-line" (p.506). Esta forma de presença é importante para o êxito do aprendiz e para o desenvolvimento de um sentimento de confiança com o administrador da faculdade.

14 Sua definição é aquela de Anderson (2007): "a medida que os participantes em uma determinada configuração de uma comunidade de investigação são capazes de construir significado através da comunicação" (citado por Kawachi, op.cit. p. 598).

15 A presença social que não é propriamente pedagógica, concerne à maneira segundo a qual o estudante se mostra aos outros através das aplicações da web social. Esta forma de presença poderia ser aproximada das identidades digitais dos internautas (Georges, 2009).

16 A presença transacional corresponde, segundo a teoria da distância transactional de Moore (op.cit.) à ruptura, nos dispositivos de formação, entre uma presença de um diálogo pedagógico (D+) e a ausência de uma estrutura imposta (S-). Kawachi se refere a definição de Shin (2002) que cita: "a disponibilidade ou a capacidade de resposta dos outros agentes envolvidos no processo educativo, como o tutor e outros alunos e a relação recíproca entre si entre cada aluno e esses outros agentes" (p.598).

170 termo é difícil de traduzir (em francês). Encontramos em numerosos textos o termo de distância pedagógica. Mas a definição de Kawachi designa antes de tudo uma forma de consciência da aprendizagem que remete à percepção do "feeling that teaching is being done" (p. 598). Eu conservarei assim o termo inglês na falta de um melhor. 
necessárias e, ao mesmo tempo, porque elas são suficientes para definir os cenários do processo de aprendizagem à distância.

A modelização proposta por Kawachi é inspirada na teoria, amplamente conhecida no mundo anglofone, mas, sem dúvida, nem tanto para os pesquisadores francofones, da distância transacional desenvolvida por Moore (1993). Relembremos rapidamente as características essenciais para compreender a contribuição do autor. A distância transacional é conhecida como um espaço fisiológico e comunicacional no qual a definição se apoia naquele mais geral do conceito de transação proposta originalmente por Dewey e Bentley em 1949. Moore utiliza por sua vez aquela, mais recente, de Boyds e Apps (1980): “a interação entre o ambiente, as pessoas e comportamentos em situação” (p. 5 apud MOORE, op.cit., p. 22). A distância transacional não é própria da formação à distância ele concerne a todos os sistemas de formação e pode mesmo contribuir para a compreensão deles. Mas à medida que ela é particularmente significativa nas situações a distância ela é mais fácil de ser analisada. Para Moore a distância transacional ${ }^{18}$ é determinada por duas variáveis, a estrutura da formação e seu grau de abertura de uma parte, e de outra, o diálogo pedagógico, as interações entre o professor e os estudantes, mas também entre esses últimos. Annie Jezégou, citando Saba e Shearer (1994), explicita o impacto dessas duas variáveis: "1) à medida que a amplitude do diálogo aumenta, a distância transacional diminui; 2) o aumento da estrutura do dispositivo diminui as possibilidades de diálogo que, por sua vez, aumentam a distância transacional" (2007, p. 11).

Na sequência do seu artigo Kawachi faz um balanço de pesquisas sobre os efeitos da presença social e discute seus resultados: notadamente as interrelações entre as distâncias sociais, cognitiva e a aprendizagem que constitui o coração da exeperiência de aprendizagem para o aprendiz, o impacto da presença social sobre a presença cognitiva, o impacto dessas distâncias, etc. Ele analisa assim

18 A distância transacional é conhecida como um espaço fisiológico e comunicacional no qual a definição se soma àquela mais geral do conceito de transação proposto na origem por Dewey e Bentley en 1949. Moore o recupera, por sua vez mais recentemente, de Boyds e Apss (1980): "the interplay among the environment, individuals and behavior in situation" (p. 5 citado por Moore op.cit., p. 22). A distância transacional não é própria à formação a distância, ela concerne todos os sistemas de formação e pode mesmo contribuir ao conhecimento deles à medida onde ela é particularmente signficativa em situações distantes. Eu já evoquei essa possibilidade de retroação no ponto 1.4 . 
as dificuldades de medir essas diferentes variáveis que demandam, como nos lembra Jézégou (2007), pouco precisas e sobretudo pouco operacionais, o que constitui, escreve ela, uma das dificuldades para validar este modelo. Entretanto, um dos aportes da contribuiçãoo de Kawachi no segundo volume de Où va la distance? (2011b) reside também na releitura crítica, na última parte do artigo, de trabalhos e de pesquisas em língua inglesa, relativas a este modelo. Eu convido o leitor interessado a ler este artigo.

\section{"A Distância: uma questão de proximidades"}

Paquelin (op.cit) usa como ponto de partida para sua reflexão a importância da presença a distância como condição de eficiência dos dispositivos de formação. Para o autor é necessário conceber a distância não como uma ruptura, mas acima de tudo, como um espaço de partilhamento e de construção social de inspiração vygotskiana. Esta maneira de teorizar a presença "destaca sobretudo as ligações, as relações entre diferentes atores (aprendizes, formadores, tutores)". Trata-se de compreender como as práticas mediatizadas de formação concorrem para esses processos de religação ${ }^{19}$ que asseguram, por sua vez, a implicação do aprendiz e a coerência e convergência do espaço-tempo social fragmentado, reconhecido como propício à realização do projeto de formação (p. 566).

Paquelin escolheu pensar a proximidade nos dispositivos de formação quer sejam eles total ou parcialmente a distância: a hibridização, do seu ponto de vista, tornou a distinção inicial entre esses modos de formação não pertinentes. Em segundo lugar, o autor se interessa aos atores, principalmente aos estudantes, à vivência de suas experiências de aprendizagem mediatizadas assim como às suas condições. Ele focaliza então concluindo o ponto de vista sobre o dispositivo apropriado e vivenciado pelo aprendiz, conhecido como um processo inscrito em múltiplas proximidades, especialmente espacial, temporal, social e cognitiva (op.cit., p. 570). Paquelin passa "de uma lógica da oferta à problemática do uso em situação” (ibidem).

O autor apresenta uma proposição original do papel das tecnologias sobre o qual ele fundamenta o potencial de proximidades no

19 Paquelin apropria-se da definição de Bolle de Bal: "a produção de relações sociais mediatizadas, o que quer dizer de relações sociais complementares onde em outros termos, a mediação de laços sociais" (2003, p.105). 
seio dos dispositivos. Eles agem como um vetor de aproximação espacial, de redução temporal e de densificação social. Eles permitem em efeito uma ampliação do território de ação que é tanto geográfico como cibergeográfico facilitando o acesso a numerosos serviços à distância, "aproximando assim o serviço de seu beneficiário" (op. cit., p. 668). Eles permitem em seguida uma redução temporal, uma imediatez à comunicação. Enfim, as tecnologias, através da religação social, multiplica a possibilidade de criar e de entreter as relações sociais com atores cada vez mais numerosos.

Finalmente, o quadro de definição das diferentes proximidades se inspira de um campo exterior àqueles das ciências da educação ou das tecnologias educativas: trata-se da análise econômica espacial desenvolvida em 1993 no campo da economia regional. Paquelin aí propõe as noções de "proximidade de essência espacial e a-espacial" (GILLES; Tore citado por PAQUELIN op.cit, p. 572) a partir das quais ele constrói um quadro de análise de dispositivos e de práticas que se desenvolvem. As diferentes proximidades espaciais concernem à localização das atividades enquanto que as proximidades espaciais revelam sua organização.

Sobre a base deste quadro teórico, ele apresenta a hipótese que essas proximidades têm um efeito benéfico para os aprendizes à medida que elas facilitam "um sentimento de autoria de seus projetos, de pertencimento e de segurança ontológica propícia ao seu engajamento no processo de formação" (op. cit., p. 566) ou ainda "o engajamento dos estudantes em um dispositivo de formação a distância supõe a ancoragem da ação em um conjunto de proximidades espaciais e a-espaciais" (op.cit., p. 566).

Dito de outra forma, essas diferentes proximidades, cujos estudos empíricos mostram que elas são interligadas, representam uma das condições da confiança dos aprendizes, de sua "segurança ontológica" e, em consequência, de sua perseverança nos dispositivos de formação.

Transações e conflito cognitivo: colaboração contraditória e presente

Annie Jézégou inscreve-se entre os trabalhos de pesquisas que tratam do modelo de distância transacional que tenta operacionalizar as variáveis, em um texto de 2007. Ela se interessa em seguida ao modelo de Garrison e Anderson (2003) para o qual ela propõe 
uma análise crítica. Em relação a esse modelo descritivo, inspirado pela perspectiva transacional de Dewey, distingue três dimensões da presença: social, cognitiva e educativa (a qual aqui associa ao papel do educador). A cada uma dessas, podemos acrescentar interações verbais particulares.

O modelo dá conta de processos de aprendizagem no sentido "comunidade de inquérito", de uma comunidade de pesquisa constituída por voluntários "de diversas áreas mas de igual valor" e caracterizada por uma demanda científica de resolução de aprendizagem colaborativa (ibidem). A autodireção e o engajamento de cada um dos membros nesta demanda constitui uma terceira característica dessa comunidade. Este modelo se apóia sobre as concepções de aprendizagem social e do socioconstrutivismo: as trocas, confrontações e negociações entre os membros, os retornos críticos e o processo reflexivo resultante dessas trocas e discussões favorecem o desenvolvimento de uma dimensão reflexiva sobre a experiência vivida, etc.

Em síntese, Jézégou sublinha o interesse desse modelo enquanto um quadro heurístico, mas ela também destaca sua principal fragilidade, a falta de definição de seus pressupostos epistemológicos e de explicitação de seus "pressupostos teóricos" como de "suas bases conceituais" (2010). Na continuação de seus trabalhos, ela propõe um modelo da presença em e-learning construída sobre uma concepção de colaboração contraditória que ela funda, do ponto de vista epistemológico, referindo-se à perspectiva transacional de ação (DEWEY, BENTLEY, 1949), assim que a corrente socioconstrutivista ancorada em psicologia social do desenvolvimento que mobiliza a teoria do conflito sociocognitivo (BOURGEOIS; NIZET, 1997; DARNON; BUTERA; MUGNY, 2008; DOISE; MUGNY, 1981; MONTEIL, 1987; PERRET-CLEMONT, 1979; PERRET-CLERMONT; NICOLE, 2-2, citados por JÉZÉGOU 2012, no $\left.1.1^{20}\right)$. Essas duas referências

sustentam juntas a concepção segundo a qual as interações sociais de conflito de negociação e de deliberação - em outros termos as transações entre os membros de um grupo para resolver de maneira conjunta uma situação problemática - têm um papel essencial na construção individual e coletiva de conhecimento [...] (ibidem, no 1.2)

20 0 Texto publicado online não está paginado. As indicações são aquelas da sub seção do texto ao qual figura o texto citado. 
Este é, brevemente relatado, o quadro teórico que fundamenta a modelização das três dimensões da presença: sociocognitiva, socioafetiva e pedagógica. A autora define a presença cognitiva como a resultante "das transações existentes entre os aprendizes que, distantes geograficamente, resolvem de maneira partilhada e conjunta uma situação problemática. As transações são de interações sociais de confrontação do ponto de vista, do ajustamento mútuo, de negociação e de deliberação que testemunham uma colaboração à distância entre os aprendizes num espaço numérico" (op.cit., 2.1).

A presença socioafetiva é definida como a resultante "de interações sociais de simetria da relação e de amenidades existentes entre os aprendizes num espaço digital de comunicação" (ibidem). Enfim, a presença pedagógica resulta "de interações sociais de coordenação de animação e de moderação que o formador entretém à distância com os aprendizes quando esses últimos, distantes geograficamente, resolvem de maneira conjunta e comunitária uma situação problemática utilizando um espaço digital de comunicação" (ibidem). Ela propõe uma representação tridimensional dessas três presenças sob a forma de três vetores que tem uma origem comum e cujo tamanho representa o valor de sua intensidade.

Jézégou sustenta que cada uma dessas presenças contribui ao desenvolvimento de uma comunidade de aprendizagem online. A presença global seria então o resultado desses vetores. A autora apresenta uma hipótese principal segundo a qual "mais a presença global é elevada, mais ela favorece o desenvolvimento de uma comunidade de aprendizagem online e, por consequência, a construção individual e coletiva e conhecimentos" (ibidem). Ela propõe em seguida, hipóteses complementares sobre as interrelações entre essas dimensões e a presença: aumento da presença sociocognitiva em razão da intensidade da presença sócio-afetiva, aumento da presença sociocognitiva em função do aumento da presença pedagógica.

Este modelo, construído com enorme rigor de sistematização até a formulação de definições -, responde às críticas feitas ao modelo de Garrisson e Anderson pelo seu esforço de ancoragem epistemológica. Ele parece extremamente sedutor, mas ele não resolve de nenhuma maneira o problema metodológico de operacionalização das dimensões, da medida quantitativa de um fenômeno qualitativo, frequentemente resolvidos em estudos desse tipo pelo uso da escala de 
Likert (KAWACHI, op. cit.). Além disso, ele deverá, segundo a própria autora ser confrontado no campo através de estudos empíricos, única maneira de se verificar a validade e a precisão das hipóteses.

A este propósito, os resultados de certas pesquisas apresentadas por Kawachi na sua revista de literatura parece confirmar as hipóteses: por exemplo, "resultados da pesquisa indicam que a presença social não tem impacto sobre a presença cognitiva de uma forma significativa" (ANNAND, 2001, p.38, apud KAWACHI, ibidem). Enfim, este modelo é o único que parece não levar em conta os dispositivos mediatizados de comunicação, salvo em termo de um contexto, não mais que seus efeitos e sua influência enquanto eles são o elemento constitutivo da aprendizagem à distância de uma parte, e enquanto eles modificam os modos de comunicação e, em consequência, as interações verbais ao centro deste modelo.

\section{Discussão}

O que assimilar/destacar dessa escolha de literatura? O que nos ensina a releitura "distanciada" desses diferentes artigos? Eu questionarei antes de qualquer coisa a diversidade de formas e de dimensões da distância, assim como da presença identificadas e diferentemente conceitualizadas a partir do questionamento inicial de Jacquinot em 1993. Eu destacarei em seguida as características principais dessa evolução, da distância à presença, que tentei colocar em evidência, sucintamente e bem imperfeitamente, com certeza.

O enfoque sobre a presença e não mais sobre a distância constitui certamente o aspecto mais importante dessa evolução. O conceito de distância e as distâncias correspondentes parecem elaboradas a partir do ponto de vista dos criadores de dispositivos de formação, eles apontam principalmente a oferta da formação, seus objetivos e suas condições de organização institucional e pedagógica. A distância é refletida numa perspectiva compensatória, como um vazio que é necessário abolir. O conceito de presença e suas presenças correspondentes são conceitualizadas do ponto de vista do uso desses dispositivos em situação de experiência de aprendizagem dos aprendizes e são decididamente voltadas para eles.

A presença, a proximidade são concebidas como um espaço digital de ação, de ligação (PAQUELIN) ou de transações, de interações 
(KAWACHI; JÉZÉGOU), entre os atores envolvidos nos dispositivos mediatizados, tendo tarefas a fazer e problemas para resolver colaborativamente. O lugar e importância dos dispositivos mediatizados parecem de igual importância quando situamos do lado da distância ou da presença. No primeiro caso, o papel dos meios é considerado como um dos fatores entre os quais todos os outros, relevam majoritariamente quadros psicopedagógicos. No modelo de Jézégou esse papel desaparece completamente: os dispositivos mediatizados constituem o quadro das interações e da presença, mais um quadro neutro, transparente, inoperante.

A meu ver, o pertencimento disciplinar dos autores e, portanto, seus quadros de referência, podem explicar essa mudança em relação aos dispositivos mediatizados. De um lado, Geneviève Jacquinot, que fez a articulação entre as ciências da informação e da comunicação de uma parte e das ciências de educação de outra, parte de um cruzamento maior desses trabalhos, se refere inteiramente ao quadro de análise das ciências de Infocom e às teorias dos meios. Por outro, do lado da presença, a filiação com as ciências da educação assim como com os fundamentos psicocoginitivos e psicossociais é evidente até se tornar dominante e exclusivo.

É coerente pensar que, nos anos 1990, o contexto favorável à EAD conduziu ao surgimento de uma reflexão sobre as dimensões da distância e da presença, assim como à eclosão dos seus diferentes modelos. Como destaque a European Association of Distance Teaching Universities Education (EADTU) como a Federação Interuniversitária de Ensino à Distância (FIED) ${ }^{21}$ são fundadas em 1987. Os numerosos relatórios oficiais franceses, suíços e europeus publica$\operatorname{dos}^{22}$ nesta época revelam bons indicadores dessa evolução. Quer eles sejam descritivos ou prospectivos, eles contribuíram enormemente para influenciar o dinamismo e renovar a pesquisa colocando em prática inúmeros dispositivos abertos e à distância. Nos anos 1990, os programas europeus de pesquisa DELTA 1 e DELTA 2 constituíram neste movimento um enorme braço de sustentação como um importante aporte financeiro para as universidades.

21 Ver sobre isso: ACHERÉ, C. (2014). História da Federação Interuniversitária do Ensino à Distância (FIED). Distances et Médiations des Savoirs, vol.6 [en ligne]. Disponível em: http://dms. Revues.org/719\#tocfrom2n20. Acesso em: 30 de nov. de 2014.

22 Citamos especialmente: CCE (1991), Enseignement supérieur ouvert et à distance dans la Communauté Européene. Rapport de la Comission, SEC (91), 897 final; CEE (1992), Memorandum sur l'apprentissage ouvert e à distance dans la Communauté Europé. 
Parece-me, portanto, que distâncias e presenças foram destacadas, identificadas e estudadas no âmbito da EAD e de suas práticas pedagógicas, mas não somente porque os contextos políticos, econômicos, tecnológicos institucionais e psicopedagógicos eram favoráveis a isso. Portanto, a maior parte dessas distâncias não caracterizam especificamente a relação pedagógica à distância: parece-me ao contrário que todo dispositivo de formação pode compartilhá-lo. Eles são transportáveis, sem dúvida com algumas adequações, aos sistemas de formação presenciais ou híbridos. Moore (1993) já destacava que a distância transacional não estava ausente dos dispositivos de formação presencial. Alguns são em efeito constitutivos do processo de ensino e de aprendizagem: é o caso das distâncias pedagógicas (JACQUINOT), cognitiva (PAQUELIN; DESSUS, et al.) e pragmática (PAQUELIN), semântica, cognitiva individual e cognitiva social (DESSUS et al.). Entretanto, os dispositivos de formação inteiramente à distância constituem o campo mais característico para compreender e analisar estes fenômenos: resta então avaliar as condições de transferência dos conhecimentos produzidos nestes contextos aos sistemas de formação presencial e híbridos (PERAYA, 1999; MEUNIER; PERAYA, 1993).

Inversamente, dois tipos de distância instrumental (FLUCKIGER, op. cit.) e epistêmica (HENRI, op.cit segundo PERAYA, 2008 e 2010) desenvolveram e conceitualizaram inicialmente e fora da relação pedagógica a distância, inicialmente sem referência ao conceito de distância. Eles primeiro identificaram e modelizaram a partir da análise dos usos das TIC, dos processos de sua apropriação em situações e do processo de gênese instrumental, relevando esferas de atividades diferentes de uma parte, e de outra, tentando compreender os efeitos desses "instrumentos", analisados em termos de mediação (PERAYA, 1999, 2010; CHARLIER et al., 2006), sobre os comportamentos dos sujeitos, quer seus efeitos sejam de natureza cognitiva (processo de produção de conhecimento) ou comunicacional (processo de difusão dos conhecimentos assim produzidos). Não é neste segundo momento que a reflexão foi transposta aos dispositivos de formação a distância, pois o uso de tecnologias assim como os processos de mediatização são inerentes ao seu distanciamento.

Eu reforçarei, assim como Jacquinot, adotando uma abordagem comunicacional dos meios e das tecnologias - as interatividades in- 
tencionais, intransitiva e transitiva - mudaram o centro de interesse da distância a uma forma de presença virtual, simulada ao âmbito mesmo do dispositivo de formação e de comunicação mediatizada. Moore, quanto a ele, analisando o diálogo pedagógico, sublinha também a importância dessa presença virtual dos interlocutores, da qual ele supunha a influência para reduzir a distância transacional:

mesmo nesses meios de comunicação (utilizados nos programas que não têm nenhuma interação) há uma forma de diálogo aluno -instrutor, porque o aluno tem uma interação interna ou silenciosa com a pessoa que em algum lugar e tempo distantes organizou um conjunto de ideias ou formação para a transmissão para o que pode ser pensado como um "diálogo" virtual, um leitor distante desconhecido, espectador ou ouvinte. [...] (JACQUINOT, 1993, p. 25).

Além disso, as teorias da presença se fundamentam sobre a análise das interações verbais mediatizadas. Podemos ignorar os efeitos dos dispositivos de mediatização sobre as formas de comunicação escrita ou verbal que elas induzem? Ou ainda, em que medida as três dimensões da presença e, em consequência, a presença global, dependem também de ambientes tecnopedagógicos como dispositivos particulares ou de sistemas de instrumentos (BOURMAUD, op.cit.) dos quais se apropriam os estudantes em situação de aprendizagem?

Enfim, todos os modelos apresentados, à exceção do de Dessus et al. (2014) que se fundamenta sobre a análise automática de distâncias semânticas, apresentam dificuldades para operacionalizar as dimensões da distância como a presença que se tornou uma realidade fundamentalmente qualitativa e contínua.

Duas direções me parecem assim se abrir hoje para a pesquisa. De uma parte, trata-se de operacionalizar e validar modelos de presença ou de proximidade como aqueles de Paquelin e de Jézégou constituem, marcos coerentes e promissores. Pode ser necessário explicitar um aspecto conceitual cujas consequências podem ser importantes: o modelo de Jézégou se distingue dos outros ao considerar a presença como um fenômeno global, composto de três dimensões, antes que formas de presença ou de proximidade distintas, cada uma apresentando eventualmente, subcomponentes estruturados e hierarquizados.

Esta segunda modelização permite identificar os fatores que determinam uma presença global e de compreender suas interrela- 
ções? A questão não é inofensiva sabendo que todos os autores, compreendidos os que trabalham a partir da distância, concordam sobre a existência dessas interrelações e que alguns tentaram deles dar conta em seus estudos empíricos (ver revista da literatura proposta por Kawachi, op.cit.). De outra parte, o exemplo de interatividades intencionais, intransitiva e transitiva mostrou, que um ponto de vista comunicacional sobre os dispositivos mediatizados podem oferecer um quadro de intelegibilidade inovador às situações de aprendizagem a distância que são em essência mediatizados. A questão, sem dúvida um pouco mais delicada, está na parte relativa que o pesquisador atribue aos dispositivos mediatizados e à importância sociocultural dos objetos técnicos (Simonian, 2014) em relação aos fatores psicológicos e pedagógicos. Em última análise a questão é aquela da interdisciplinaridade e da articulação entre as ciências da Educação e as da Informação e da Comunicação.

\section{REFERÊNCIAS}

AUDET, L.. Regards sur l'évolution de la formation à distance au Canada francophone. In: Guillemet P., Fichez E., Barna J. \& Vidal M., dir., Distances et savoirs, Où va la distance ? Tome 1, vol. 9, n 3, 2011, p. 313-330. Disponível em: ligne : http://www.cairn.info/revue-distances-et-savoirs.htm DOI : $10.3166 /$ ds.9.313-330

BARCHECHATH, E. \& Pouts-Lajus, S.. Sur l'interactivité. In: CROSSLEY, K., GREEN. L. Le design des didacticiels : guide pratique pour la conception de scénarios pédagogiques interactifs, Paris, ACL éditions, 1990.

BOLLE, de Bal M. Reliance, déliance, liance : émergence de trois notions sociologiques. Sociétés, vol. 2, nº 80, 2003.

BOURDMAUD, G.. Les systèmes d'instruments : méthodes d'analyse et perspectives de conception (Thèse de doctorat sous la direction de P. Rabardel), Paris Saint Denis, Université de Paris 8, 2006. Disponível em ligne :http://www. bibliotheque-numerique-paris8.fr/fre/notices/103373-Les-syst\%C3\%A8-mes-dinstruments-m\%C3\%A9-thodes-d-analyse-et-perspectives-de-conception.html

CHARLIER, V., DESCHRYVER, N. \& PERAYA, D. Apprendre en présence et à distance : une définition des dispositifs hybrides. Distances et savoirs, vol. 2, $\mathrm{n}^{\circ}$ 4, 2006, p. 469-496. Disponível em: http://www.cairn.info/revue-distances-etsavoirs-2006-4-page-469.htm(consulté le 19 novembre 2014).

DOI : $10.3166 /$ ds.4.469-496 
CHARLIER, B. \& HENRI, F. Apprendre avec les technologies. Paris, PUF, 2010. Disponível em : 10.3917/puf.charl.2010.01

CHRISTENSEN, G.; STEINMETZ, A.; BENNET, A; WOODS, D. \& EMANUEL, E.J. The MOOC Phenomenon: Who Takes Massive Open Online Courses and Why? Université de Pennsylvanie, 2013. Disponível em :http://papers.ssrn.com/ sol3/papers.cfm?abstract_id=2350964 (mis en ligne le 18 avril 2014) (consulté le 19 novembre 2014).

CLARK, H.H. \& BRENNAN, S.E. Grounding communication. RENICK, L.B.; LEVINE, J.L.; \& TEASLEY, S.D (org.) In: Perspectives on socially shared cognition, Washington, American Psychological Association, 1991.

DAFT, R.L. \& LENGEL, R.H. Information richness: A new approach to managerial behaviour and organization design, Research in Organizational Behavior, n 6, p. 191-233, 1984.

DENNIS, A.R., \& VALACICH, J.S. Rethinking Media Richness: Towards a Theory of Media Synchronicity. In: Proceedings of the 32nd Hawaii International Conference on System Sciences. Conference on System Sciences-Volume 1, Washington, IEEE Computer Society, p. 1017.

Disponível em: 10.1109/HICSS.1999.772701

DESSUS, P., TRAUSAN-MATU, S., WILD, F., DUPRÉ, D., LOISEAU, M., REBEDA, T. \& ZAMPA V. Un environnement personnel d'apprentissage évaluant les distances épistémiques et dialogiques. In: GUILLEMET, P., FIXCHEZ, E., BARNA, J. \& VIDAL, M., (org.), Distances et savoirs, Où va la distance ? Tome 2, vol. 9, n 4, p. 473-492, 2012. Disponível em: http://www.cairn.info/revue-distanceset-savoirs.htm (consultado 19 novembro 2014).

DUSSARPS, C. Dimension socio-affective et abandon en formation ouverte et à distance (Thèse de doctorat sous la direction de D. Paquelin), Bordeaux, Université de l'Université Montaigne, 2014.

FLUCKIGER, C. L'analyse des Environnements Personnels d'Apprentissage sous l'angle de la discontinuité instrumental. STICEF. Les environnements personnels d'apprentissage, entre description et conceptualisation, 2014. Disponível em : http://sticef.org

FLUCKIGER, C. De l'émergence de nouvelles formes de distances. Les compétences des nouvelles pratiques de communication ordinaire sur la FAD dans le supérieur. In: GUILLEMENT, P.; FICHEZ, E., BARNA, J. \& VIDAL, M., (org.) Distances et savoirs, Où va la distance ? Tome 1, vol. 9, n 3, p. 397-417, 2011. Disponíel em: http://www.cairn.info/revue-distances-et-savoirs.htm (acesso 19 novembro 2014).

GEORGES, F. Représentaion de soir et identité numérique, Une approche 
sémiotique et quantitative de l'emprise du web 2.0. Réseaux, vol. 2, nº 154, p. 165-193, 2009.

GLIKMAN, V. Des cours par correspondance au "E-learning”, Paris, PUF, 2002.

GUILLEMET, P; FICHEZ, E; BARNA, J. \& VIDAL, M; (org.) Distances et savoirs, Où va la distance ? Tome 1, vol. 9, n 3, 2011a. Disponível em:http:// www.cairn.info/revue-distances-et-savoirs.htm (consulté le 19 novembre 2014.

GUILLEMET, P; FICHEZ, E; BARNA, J. \& VIDAL, M; (org.) Distances et savoirs, Où va la distance ? Tome 2, vol. 9, n 4, 2011b. Disponível em :http:// www.cairn.info/revue-distances-et-savoirs.htm (acesso 19 novembro 2014).

GUIR, R; (org.) Pratiquer les TICE. Former les enseignants et les formateurs à de nouveaux usages. Bruxelles, De Boeck, 2012.

HeENRI, F. \& KAYE, A. Le savoir à domicile. Pédagogie et problématique de la formation à distance. Québec, Presses de l'Université du Québec, 1985.

HeENRI, F. Où va la distance ? Est-ce la bonne question. GUILLEMET, P; FICHEZ, E; BARNA, J; VIDAL, M. (org.) Distances et savoirs, Où va la distance ? Tome 2, vol. 9, n 4, 2011, p. 619-630. Disponível em :http://www.cairn.info/revuedistances-et-savoirs.htm (consulté le 19 novembre 2014).

HOUSSAYE, J. Le triangle pédagogique, Berne, Peter Lang, 1988.

JACQUINOT, G. Apprivoiser la distance et supprimer l'absence ? ou les défis de la formation à distance. In: Revue française de pédagogie, $\mathrm{n}^{\circ} 102$, p. 55-67, 1993. Disponível em : 10.3406/rfp.1993.1305

. Qu'apporte l'interactivité à l'apprentissage ? CRINON, J;

GAUTELIER, C; (org.)In: Apprendre avec le multimédia, où en est-on?, Paris, Retz, 1997.

Absence et présence dans la médiation pédagogique ou comment faire circuler les signes de la présence. GUIR, R. (org.) Pratiquer les TICE. Former les enseignants et les formateurs à de nouveaux usages, Bruxelles, De Boeck, p. 104113.

JÉZÉGOU, A. La présence en e-learning modèle théorique et perspective pour la recherche. In: La Revue internationale de l'apprentissage en ligne et de l'enseignement à distance (IJEDE), vol. 26, n 1, 2012. Disponível em :http://www. ijede.ca/index.php/jde/article/view/777/1409 (acessado 19 novembro 2014).

. Community of Inquiry en e-learning à propos du modèle de Garrison et d'Anderson. In: La Revue internationale de l'apprentissage en ligne et de l'enseignement à distance (IJEDE), vol. 24, n² 2, 2010, p. 1-18. Disponível 
em : http://www.ijede.ca/index.php/jde/article/view/652/1102(acessado 19 novembro 2014).

La distance en formation. Premier jalon pour une opérationnalisation de la théorie de la distance transactionnelle. Distances et Savoirs, vol. 5, $\mathrm{n}^{\circ} 3$, 2007, p. 341-366. Disponivem em :http://www.cairn.info/revue-distances-etsavoirs-2007-3-page-341.htm(acessado 19 novembro 2014).

DOI : $10.3166 /$ ds.5.341-366

KAWASHI, P. Unwrapping presence. GUILLEMET, P; FICHEZ, E; BARNA, J. \& VIDAL, M. (org.) In: Distances et savoirs, Où va la distance? Tome 2, vol. 9, $\mathrm{n}^{\circ} 4$, p. 591-606, 2001. Disponível em : http://www.cairn.info/revue-distances-et-savoirs. htm (acessado 19 novemrbo 2014).

LYOTARD, J.F. La condition post-moderne. Rapport sur le savoir. Paris, Minuit, 1979.

MEUNIER, J.-P. \& PERAYA. Introduction aux théories de la communication. Analyse sémio-pragmatique de la communication médiatique. Bruxelles : De Boeck, (2e édition revue et augmentée, 2004), 1993.

MOORE, M. Theory of transactional distance. In: KEEGAN, D. Theoritical principles of distance education, Londres et New York, Routledge, 1993.

PAQUELIN, D. La distance : question de proximities. GUILLEMET, P; FICHEZ, E; BARNA, J. \& VIDAL, M. (org.) In: Distances et savoirs, Où va la distance? Tome 2, vol. 9, n 4, 2011, p. 565-589. Disponível em :http://www. cairn.info/revue-distances-et-savoirs.htm (acessado 19 novembro 2014). DOI : 10.3166/ds.9.565-590

Présence, distance : vers de nouvelles configurations

organisationnelles. In: Distances et Médiations des Savoirs, $\mathrm{n}^{\circ}$ 7, 2014. Disponível em: http://dms.revues.org/797 (acesso 19 novembro 2014).

DOI : $10.4000 / \mathrm{dms} .797$

PERAYA, D; CHARLIER, B. \& DESCHRYVER, N. Une première approche de l'hybridation. Education et formation. Les dispositifs hybrides dans l'enseignement supérieur : questions théoriques, méthodologiques et pratiques, vol. e-301, 2014, p. 15-34. Disponível em :http://ute3.umh.ac.be/revues/include/download. php?idRevue=19\&idRes=183(acessado 19 novembro 2014).

PERAYA, D. Quel impact les technologies ont-elles sur la production et sur la diffusion des connaissances? In: Questions de communication, vol. 21, 2012, p. $89-106$.

DOI : $10.4000 /$ questionsdecommunication. 6590 
Un regard de la "distance" vue de la "présence”. GUILLEMET, P; FICHEZ, E; BARNA J. \& VIDAL M; (org.) In: Distances et savoirs, Où va la distance? Tome 1, vol. 9, n 3, 2011, p. 445-452. Disponível em :http://www.cairn. info/revue-distances-et-savoirs.htm (acessado 19 novembro 2014).

. Médiatisation et médiation. Des médias éducatifs aux ENT. In: LIQUÈTE, V; (org.) Médiations, Paris, CNRS, 2010, p. 35-48.

. Technologies, mutations des connaissances et de l'apprentissage : impact sur les métiers d'enseignant et d'étudiant. BERGADAÀ, $M$; DELLAMBROGIO, P ; FALQUET, G., MCADAM, D ; PERAYA, D. \& SCARIATI, $\mathrm{R}$. In : La relation éthique-plagiat dans la réalisation des travaux personnels par les étudiants. Rapport 2008, Commission Ethique-Plagiat, Genève, Université de Genève, 2008, p. 19-31. Disponível em : http://archive-ouverte.unige.ch/ unige:6338 (Acessado 19 novembro 2014).

Médiation et médiatisation : le campus virtuel. Hermès, $n^{\circ} 25,1999$, p. $153-168$. DOI : $10.4267 / 2042 / 14983$

POMEROL, J.C. Les universités à l'heure des MOOC, Contribution à la conférence. Les universités à l'heure des MOOC. Printemps UNI_UNF3S ». 3 et 4 juin 2014. Disponível em :http://www.printempsunt2014.univ-lorraine. fr/files/2014/07/pr\%C3\%A9sentation-MOOC-pomereol.pdf (consulté le 19 novembre 2014).

SALOMON, G. Interaction of Media, Cognition and Learning, San Francisco, Jossey-Bass, 1999.

SIMONIAN, S. L'affordance socioculturelle : une approche écoanthropocentrée des objets techniques. Le cas des environnements numériques d'apprentissage (mémoire d'Habilitation à diriger les recherches sous la direction de B. Albéro), Rennes, Université de Rennes 2, 2014. 